\title{
Amplitude measures of operant response: Implementation with Apple Pascal
}

\author{
STEPHEN C. FOWLER \\ University of Mississippi, University, Mississippi
}

\begin{abstract}
Because operant conditioning studies involving force of response as a dependent variable require high-speed A/D conversion, conventional operant software, which treats the input datum as a dimensionless event, is generally unsuited for this kind of work. The author describes a complete Apple-based hardware-software ensemble that measures and records on diskette the peak force, duration, time integral of force, and interresponse time for individual operant responses. Data acquisition and control programs were written in Apple Pascal linked to selected assembly language modules. The real-time base was derived from an inexpensive crystal clock interfaced through the game port.
\end{abstract}

A wide variety of hardware and software configurations are available for processing the digital inputs (switch closures) from which conventional operant conditioning measures are derived (e.g., Gordon, Foree, \& Eckerman, 1983). However, once interest turns to the force of operant responding, the availability of microcomputer solutions to this measurement problem is scarce. Fowler (1974) described a PDP-12 minicomputer system for conducting operant research in which response force receives the scrutiny usually devoted to response rate. Since the PDP-12 technology is now obsolete, the purpose of this paper is to outline an inexpensive Apple-based system that duplicates the force measurement concepts used by Fowler (1974) for digital systems and by Notterman and Mintz (1965) for an analog computer system.

The software described herein was written predominately in Pascal, along with a few assembly language routines. Pascal was chosen because of several features that make it more suitable than BASIC for real-time work. Pascal, a compiled language, is faster than BASIC (Apple Pascal is compiled into an intermediate p-code, not native machine language; nevertheless, it is still much faster than BASIC); the UCSD p-system supplied by Apple Computer, Inc., makes the mixing of assembly code and Pascal code exceedingly easy. Pascal is much more efficient than BASIC in terms of programmer effort. Pascal code is easier than BASIC to decipher and modify. And Pascal data structures facilitate diskette I/O operations. Primary disadvantages of Pascal are that it is more difficult than BASIC in the initial stages of learning and Pascal does not reside in firmware as does BASIC. However, the advantages far outweigh the disadvantages, especially in real-time, process-control applications.

Software needed for the measurement of the quantitative properties of individual operant responses may be

The author is affiliated with the Departments of Psychology and Pharmacology. His mailing address is: Department of Psychology, University of Mississippi, University, MS 38677. divided into three functional categories: calibration, data acquisition-process control, and data reduction/display. Programs to accomplish each of these functions will be described in turn, but it will first be necessary to give an overview of the required hardware with some additional detail for the real-time base.

\section{HARDWARE}

The minimum hardware configuration includes a $64 \mathrm{~K}$ Apple II +, Apple Ile, or Franklin 1000 microcomputer, one disk drive, a monitor, an analog-to-digital (A/D) converter, a real-time clock, an output interface, and a suitable force transducer with associated signal-conditioning circuitry. Although one disk drive is sufficient to execute the programs, two drives are almost mandatory for easy software modification. Moreover, the programs themselves require considerable diskette space, and as a result, the amount of space available for data is quite limited in a one-drive system. The assembly language procedure used by the data-acquisition program assumes that a John Bell A/D converter (Model 81-132, John Bell Engineering, 1014 Center St., San Carlos, CA 94070) is located in peripheral slot 5 . Table 1 gives the assembly language code used to service this A/D converter. See FUNCTION SAMPLEB in Table 1. The real-time base and output signals are handled through the game port, which is conveniently accessed through Apple Pascal's Applestuff utility or through 6502 assembly language procedures such as TIMEYET, REINFRCE, or STEPPER, listed in Table 1 . The hardware/software system described here can service only one force transducer; however, additional real-time performance can be achieved by adding a SpeeDemon board to one of the Apple peripheral slots. This device speeds processing by a factor of 3.5 and works with the Apple Pascal software without modification. Use of the SpeeDemon should make it possible to service two transducers with a single computer. The SpeeDemon is available from MCT, 174521 st St., Santa Monica, California 90404 . 
Table 1

Assembly Language Procedures Called By the Pascal Data-Acquisition and Control Programs

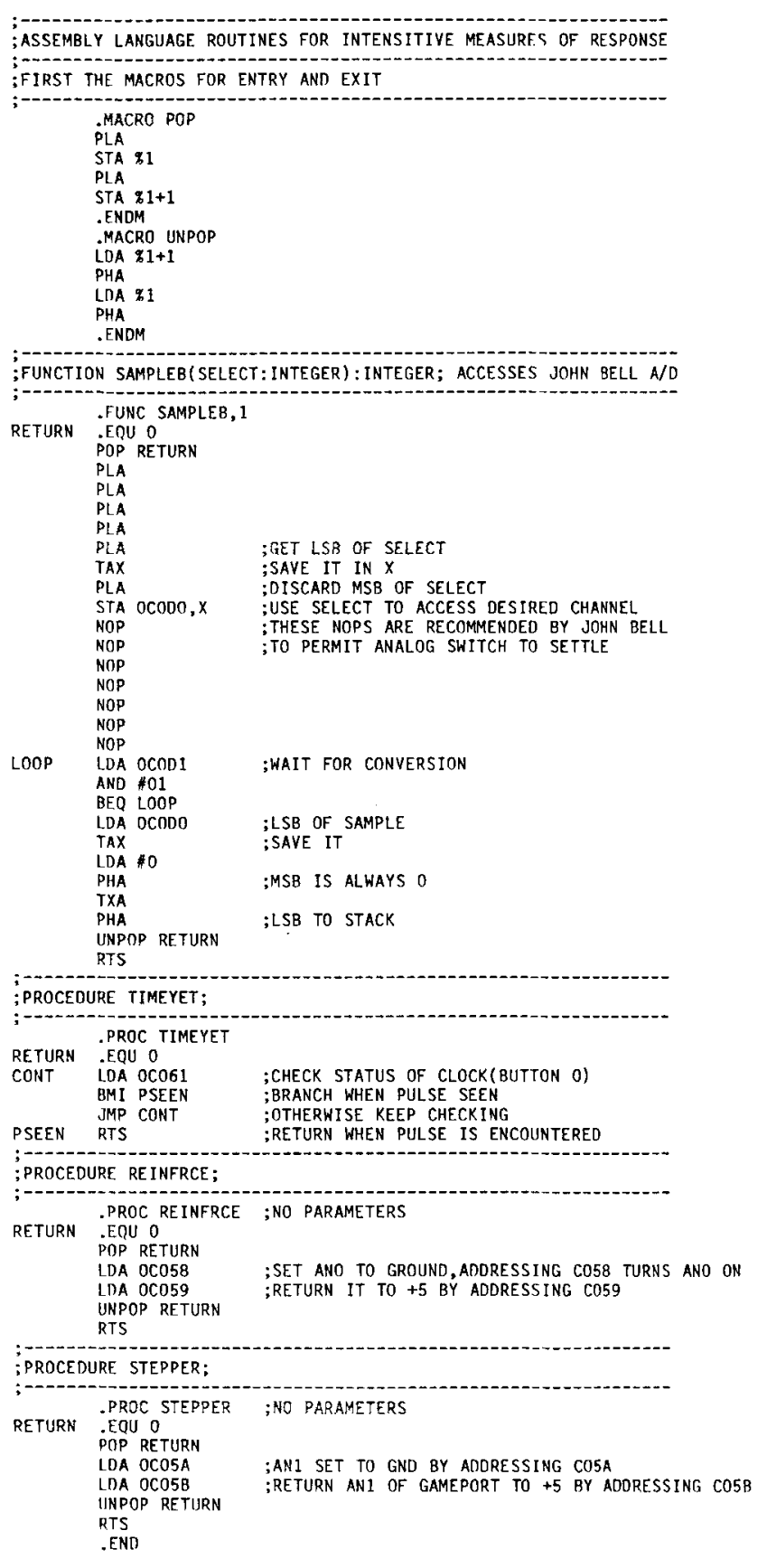

One straightforward method for interfacing the TTL outputs of the game port to external equipment (i.e., relays, feeder solenoids, etc.) is described in Fridlund, Price, and Fowler (1979), and the software delineated here makes use of this "buffer/driver" circuit. Instead of using a commercial product for the clock, the system relies on an inexpensive home-brew time base (see Figure 1). The clock can be mounted on a proto board, bringing the total cost of the clock to about $\$ 35$.

Table 1 lists a simple assembly language procedure (coded as a Pascal procedure without parameters) for detecting the clock pulses. The three-instruction loop in TIMEYET, consisting of LDA 0C061, BMI PSEEN, JMP CONT repeatedly examines the status of game-port button 0 ; upon detection of the timing pulse, control returns to the host program. One reason for keeping the clock pulse at $20 \mu \mathrm{sec}$ is to ensure that the same clock pulse is not sensed twice, which could happen with a longer pulse if the TIMEYET procedure was rapidly called again. Another reason for selecting the brief 20 $\mu \mathrm{sec}$ "on" time is to provide a large amount of time (compared with a $50 \%$ duty cycle) between clock pulses to accommodate some of the longer processing loops in the host data acquisition and control program.

To combine Pascal programs with assembly language modules, such as those shown in Table 1, one must link together the respective code files to form a functioning whole. In preparing the Pascal source code, the user refers to the assembly language procedures or functions by name and identifies these with an external declaration. For example, FUNCTION SAMPLEB shown in Table 1, is listed in the Pascal source code as "FUNCTION SAMPLEB(SELECT:INTEGER):INTEGER; EXTERNAL;". Thus, SAMPLEB has one parameter that is an integer type, and the value of the function is itself an integer type. During compilation of the Pascal source, the EXTERNAL directive indicates to the compiler that SAMPLEB will be supplied as a machine language module in a later-tobe-performed linking operation. The single parameter of SAMPLEB is the A/D channel that is to be sampled, and the value of SAMPLEB itself is the digitized value. Once the Pascal source has been compiled, the user runs the SYSTEM.LINKER and specifies the just-compiled Pascal code file as the "host" file and the assembly language code file as the "lib" (for library) file. In the present case, the assembly language in Table 1 was stored in a source file named BITLEVEL.TEXT. This file was submitted to the SYSTEM.ASSEMBLER that produced the code file BITLEVEL.CODE. Output of the LINKER is the desired executable code file with the assembly language routines incorporated into the Pascal host file.

Those familiar with Apple Pascal will notice that the three procedures TIMEYET, REINFRCE, and STEPPER could have been implemented through BUTTON or TTLOUT procedures contained within the Applestuff utility. These utilities were not used in order to save time; both BUTTON and TTLOUT require stack manipulation upon entry and exit and, therefore, consume many machine cycles. However, the parameterless procedures in Table 1 require no manipulation of the stack and as a result execute faster than their Applestuff counterparts.

An alternative interfacing method uses the Apple interface board (Model 530AI with A/D and clock options in- 


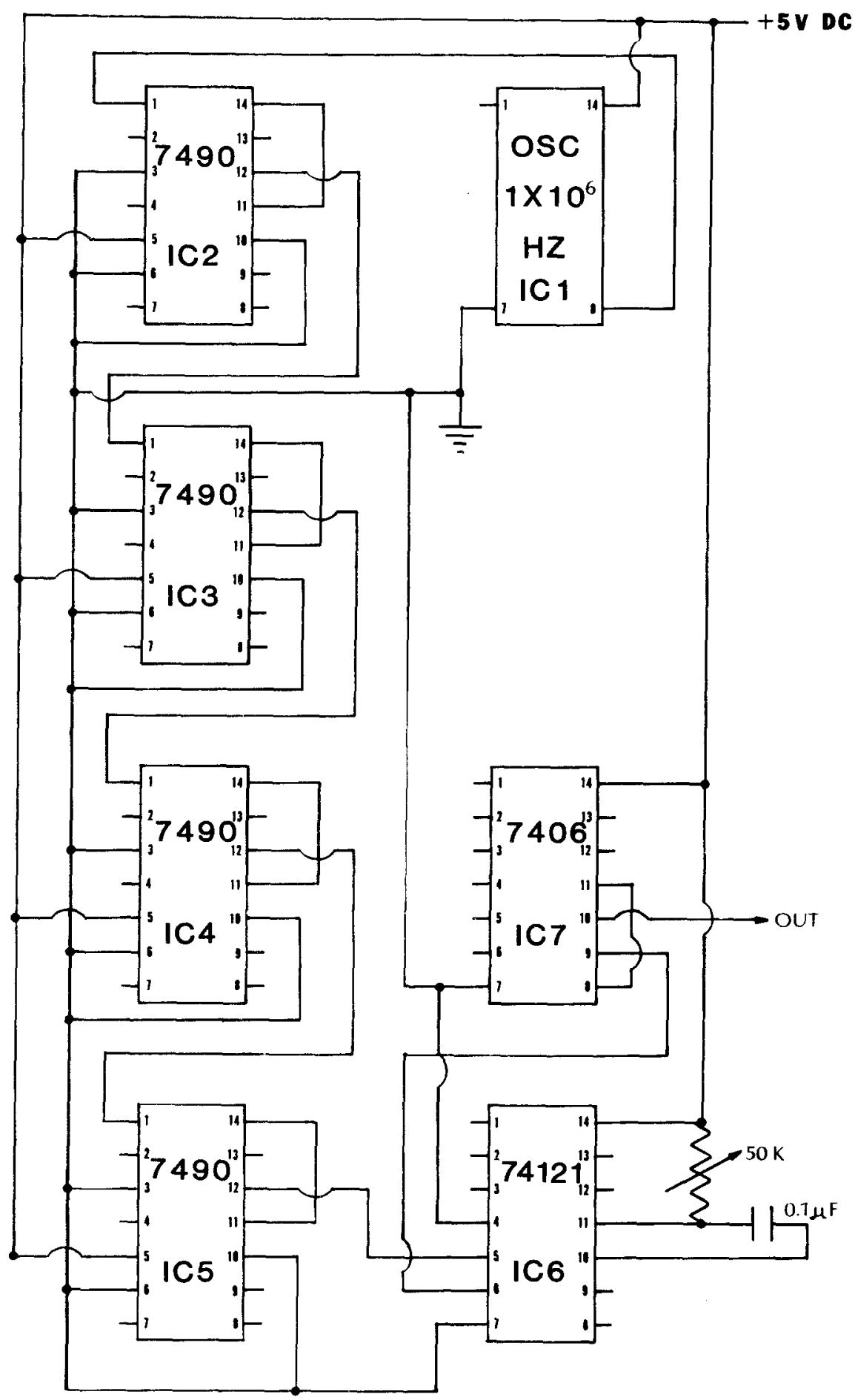

Figure 1. An inexpensive, crystal-controlled, $100-\mathrm{Hz}$ clock whose output is used to drive a button input on the Apple game port. The clock consists of a 1-MHz, modular, TTL-compatible square-wave oscillator whose output is divided by 10,000 through the use of 7490 decade dividers. The resultant $50 \%$ duty cycle square wave is used to trigger a 74121 monostable multivibrator to produce a 20 - $\mu$ sec pulse every $0.01 \mathrm{sec}$. A 7406 supplies sufficient buffering and drive capacity for three computers to share one clock. The timing pulse activates one of the button inputs on the Apple game port. Although not shown in Figure 1, the button input terminal must be held high through a $680-\Omega$ pull-up resistor. Software monitors the status of the clock-button input to detect the occurrence of the 20- $\mu$ sec pulse; thus, each pulse marks the end of a 0.01-sec period. 
stalled) supplied by Life Science Associates, One Fenimore Rd., Bayport, New York. Moreover, the software described herein will be available in 1985 from Life Science Associates (LSA). The primary differences between the two approaches are that the $530 \mathrm{AI}$ board uses a $62.424-\mathrm{Hz}$ clock instead of the $100 \mathrm{~Hz}$ described here, and the LSA version employs software to time the duty cycles of stepper and reinforcer, whereas the buffer/driver used here performs this timing in external hardware.

\section{SOFTWARE}

From a functional perspective, the software provides for system calibration, data acquisition-process control, and data reduction/display. Programs that carry out these functions are briefly described in this section. Readers unfamiliar with the measurement concepts used in operant force studies will find it helpful to read the paper by Fowler (1974).

\section{Calibration}

Two separate kinds of system calibration need to be considered: time calibration and force calibration. Since preservation of the real-time base is necessary for meaningful measurements, and since one cannot conveniently determine in advance whether one's Pascal code will execute as fast as desired, it is important to check the system's performance against precisely calibrated input signals. If the user has a precision frequency source, this procedure is straightforward. By using the data-acquisition software (described below) to collect data from the frequency standard, the input can be compared with the output. For the time calibration, a square-wave frequency source can be used, but some form of amplitude adjustment must be employed to test the system both for suprathreshold response amplitudes and for supracriterion response amplitudes. For example, with the frequency source amplitude set at a voltage above the threshold but below the criterion, a $10-\mathrm{Hz}$ square-wave ( $50 \%$ duty cycle) signal is fed into the acquisition-control program. Under these conditions, the program should report response durations of $0.05 \mathrm{sec}$ and interresponse times (IRTs) of $0.05 \mathrm{sec}$, with uniform, subcriterion peak force values. The amplitude of the frequency source should then be set to a value above the force criterion voltage, with the frequency again at $10 \mathrm{~Hz}$. This portion of the time calibration test forces the program through the responseconsequences loops. As in the previous example, the program should report response durations of $0.05 \mathrm{sec}$ and IRTs of $0.05 \mathrm{sec}$, but the peak force values recorded should be consistent at the selected supracriterion value. Duration or IRTs of less than $0.05 \mathrm{sec}$ in the second test are evidence that the program loops require more than $0.01 \mathrm{sec}$ for execution. Time calibration needs to be performed after any modifications of the Pascal software that involve the addition of one or more instructions anywhere within the real-time branches of the program.
In performing calibration using a conventional operant apparatus, the integrity of the response-sensing microswitch is easily ascertained: The experimenter simply operates the switch to verify that the system is functioning. However, when a force transducer is used as the operandum, the calibration procedure is somewhat more involved. The investigator must check the electrical zero point, linearity, and the sensitivity of the instrument. Two separate Pascal programs are available for accomplishing routine calibration of the force transducer (or any other quantity expressed as a voltage analog). These are SCO$\mathrm{PEX}$ and CALIBX. By digitizing 100 samples/sec and displaying 260 samples in high-resolution graphics, SCOPEX performs some of the functions of an oscilloscope. The visual display of data permits one to set a zero point and to observe the amount of electrical interference caused by $60-\mathrm{Hz}$ radiation, transients from solenoids, etc. Moreover, the transducer's natural frequency oscillations (or "aliases" thereof) can be visually detected with this program. (For a discussion of natural frequency oscillations in the context of response force measurement, see Fowler, in press.) Whereas SCOPEX provides for a relatively gross evaluation of the transducer signal, CALIBX enables the operator to check numerically the sensitivity and linearity of the instrument. This program has an averaging routine so that calibration can be accomplished even if some electrical noise is present in the input signal. Typically, the calibration procedure involves placing weights on the transducer and adjusting zero and gain controls on the transducer amplifier/conditioner until satisfactory measurements are displayed on the monitor by CALIBX.

\section{Data Acquisition-Process Control}

The Pascal program for collecting data and appropriately dispensing scheduled events is named FORCE. It permits the user to specify any one of the five simple schedules of reinforcement: fixed ratio, variable ratio, fixed interval, variable interval, and differential reinforcement of low rates. Specification of parameters for an experimental session is menu driven so that the user may select schedule of reinforcement, schedule parameters, force parameters, and conditions for terminating a session. The latter may be based on either elapsed time or number of responses emitted. The file name under which the data are to be stored is also entered during the startup dialog.

FORCE samples the transducer voltage every $0.01 \mathrm{sec}$ and determines whether the obtained sample is above or below the threshold for defining a response. Typically, the threshold is $4 \mathrm{~g}$, a value low enough to register almost every contact of a rat's paw with the operandum but high enough to make the system unresponsive to extraneous vibrational or electrical events. The threshold force is specified during the start-up dialog. The threshold is the same for defining both the beginning and the end of a response; hysteresis (e.g., $4 \mathrm{~g}$ to begin the response and $3 \mathrm{~g}$ to end the response) is not needed because force trans- 
ducers suitable for measuring response force do not exhibit appreciable hysteresis. The transducer's natural frequency oscillations analogous to switch contact bounce are not easily handled by software, and such oscillations are usually removed by electronic filtering before the signal arrives at the A/D converter (Fowler, in press).

Once the force proportional voltage exceeds the threshold, the program begins taking the measurements for the response in progress. These values, recorded for every response, are peak force, duration, and time integral of force. As its name indicates, peak force is simply the maximum force value attained. Duration is the amount of time the response force remains above threshold, and time integral of force is the area under the force-time waveform. Since each response has a specified beginning and end, the IRT is explicitly separated from response duration; that is, with this measurement system, IRT is taken as the time from the end of one response to the beginning of the next response. Unlike the system presented here, many microswitch-based operant systems define the IRT as the interval between the beginning of one response (lever depression) to the beginning of the next response. In addition to collecting the four dependent variables (three for the response itself and one for the time between responses), the program determines, upon response termination, whether the just-ended response qualifies for reinforcement. In cases in which a criterion other than response occurrence is used, the program compares the
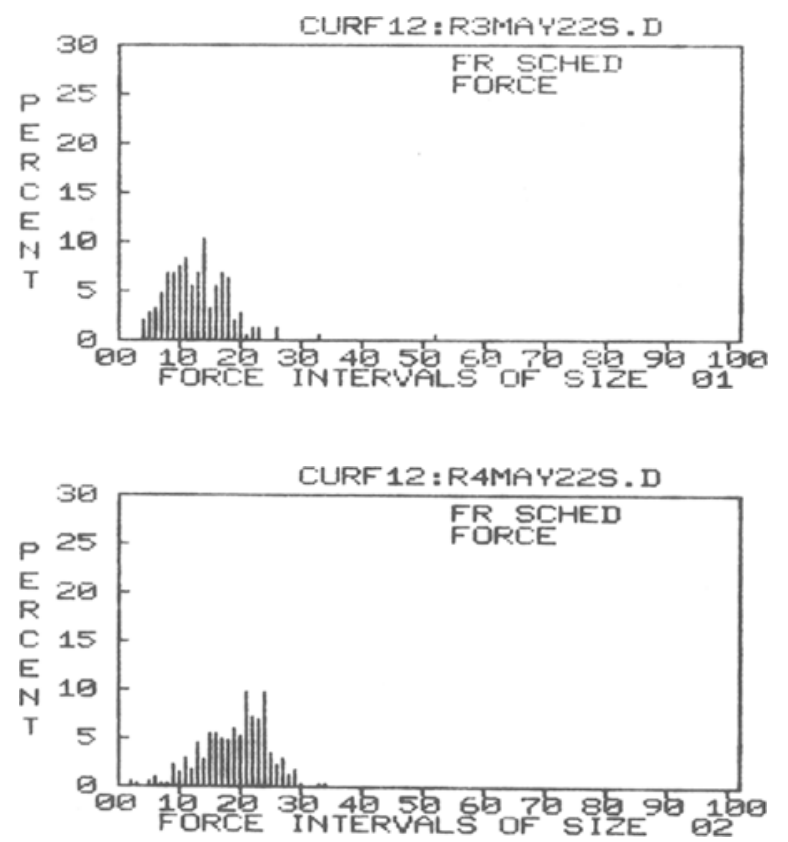

criterion variable against the criterion constant specified during the start-up dialog. For example, if the peak force criterion is $40 \mathrm{~g}$, the program will not produce scheduled consequences unless the response attains a peak force of $40 \mathrm{~g}$ or more. Procedurally, it is possible to specify the criterion in terms of any one of the four response properties measured by the computer. However, current versions of the software use only peak force as the criterion quantity, except, of course, for the DRL schedule, which permits specification of criterion IRT as well as peak force. Because the bulk of the software is written in Pascal, one can change the criterion dimension by modifying only one or two instructions.

In addition to recording the four dependent variables for each response, the program logs the elapsed time (to the nearest second) between the start of a session and the emission of a particular response. Also indicated in the data array is the occurrence or nonoccurence of response consequences, such as reinforcement delivery.

Upon completion of an operant session, which can be terminated on the basis of session time or number of responses emitted, the computer's speaker makes a series of musical sounds and the data stored in RAM are written to a diskette file, whose name was specified during the start-up dialog. Appended to the end of the data array for individual response measurements is session summary information consisting of peak force threshold, peak force criterion, schedule of reinforcement, number of re-
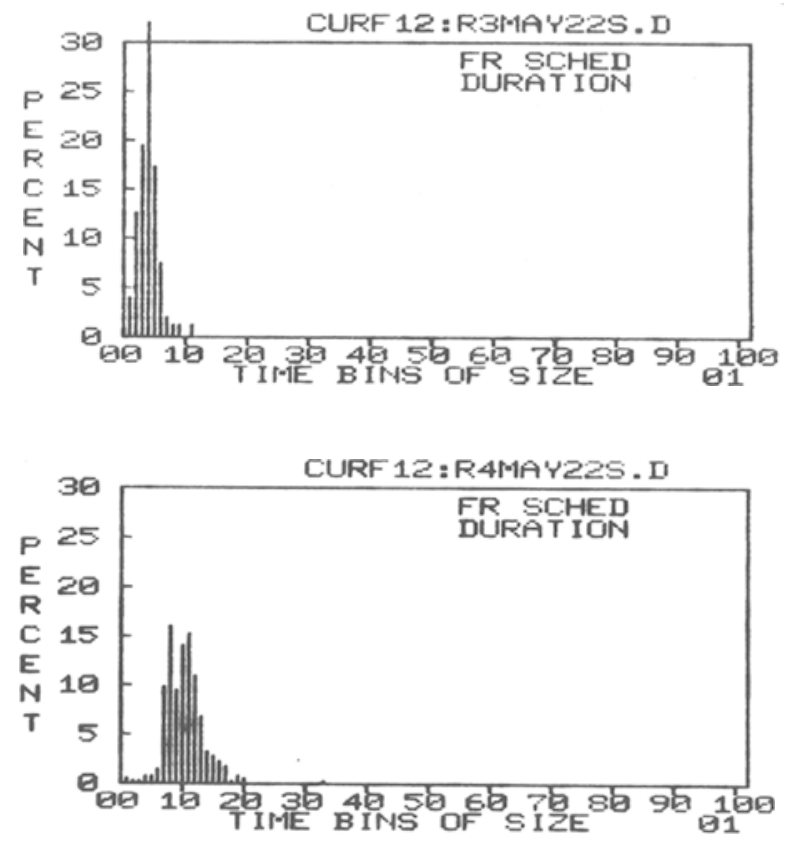

Figure 2. Relative frequency distributions of peak force and duration of operant response for two rats responding for water on a continuous reinforcement schedule. For $R 3$ (top two axes), the peak force criterion was $4 \mathrm{~g}$; for $\mathbf{R} 4$ (bottom two axes), the peak force criterion was $40 \mathrm{~g}$. For R3, data are for 143 responses; for R4, they are for 353 responses. Note the difference in force scales for the two rats; that is, the bin size in the lower force plot is $2 \mathrm{~g}$, but in the upper force plot is $1 \mathrm{~g}$. These plots were produced by dumping Apple I+ high-resolution images to an Epson FX80 via a PKASO parallel interface. 
sponses, number of reinforcers, and session time. The global session data are then available on diskette for use by the data analysis programs.

\section{Data Reduction and Display}

Since even the simplest behavioral procedures produce several thousand measurements per rat per experimental session, some degree of data reduction is required in order to characterize parsimoniously a rat's performance. Two programs have been written for this purpose. The first, named FANAL, calculates the means and standard deviations for each of the four dependent variables. FANAL also obtains the Pearson correlations among these variables. These statistics, along with the session summary data, average response rate, and the file name (to identify the subject and experimental conditions) are transferred to a printer.

Taking advantage of Apple Pascal's high-resolution graphics capabilities, a second program, named FREQFORC, compiles and displays relative frequency distributions for each of the four dependent variables. Examples of output, dumped onto an Epson FX-80 printer via a PKASO interface, are shown in Figure 2. For the sake of brevity, only peak force and duration are presented here. These data are for two separate rats (R3 and R4; note the two characters following the colon in the labels just outside the axes). $R 3$ received 3-sec access to water for every response of $4 \mathrm{~g}$ or more, whereas the peak force criterion for R4 was $40 \mathrm{~g}$. Medians (also calculated by FREQFORC) for the two peak force distributions were 13.2 and $41.1 \mathrm{~g}$ for R3 and R4, respectively. For the corresponding response duration distributions (right half of Figure 2), the medians were 0.088 and $0.107 \mathrm{sec}$ for $\mathrm{R} 3$ and $R 4$, respectively. The frequency distribution program has some flexibility in scaling the $x$ and $y$ axes. For instance, the peak force distribution for R4 in Figure 2 (lower axes) is scaled so that each vertical bar represents the percent of responses in a bin $2 \mathrm{~g}$ wide, whereas the interval size for $\mathbf{R} 3$ (upper axes) is $1 \mathrm{~g}$. Routine exami- nation of these frequency distributions has been a valuable source of information in helping the experimenter decide what statistic best describes the effects of independent variables on the four response measures.

Both data analysis programs allow the user to key in the names of several (up to 80) data files. The input routine features an editing function that permits the user to correct any typing errors before submitting the files for analysis. Thus, the programs can process a large number of data files without operator intervention.

\section{SUMMARY}

The once-difficult task of measuring the quantitative properties of individual operant responses (peak force, duration, etc.) has been greatly simplified by making use of Apple Pascal. The Apple-based UCSD Pascal system makes it very easy for one to mix Pascal procedures with assembly language modules to achieve the highperformance, yet inexpensive, data acquisition and analysis system described herein. Moreover, the transportability of Pascal should provide for relatively straightforward implementation of these response-measuring techniques on microcomputers other than the Apple II family of machines.

\section{REFERENCES}

Fowler, S. C. (1974). A minicomputer system for recording the dynamic properties of individual operant responses. Behavior Research Methods \& Instrumentation, 6, 288-292.

FowLER, S. C. (in press). Force and duration of operant response as dependent variables in behavior pharmacology. In T. Thompson, P. B. Dews, \& J. E. Barrett (Eds.), Advances in Behavioral Pharmacology. Fridlund, A. J., Pruce, A. W., \& Fowler, S. C. (1979, November). The output buffer driver: An interface that provides DC switching of multiple external devices. Kilobaud-Microcomputing, pp. 146-150.

GoRDON, W. A., Foree, D., E Eckerman, D. S. (1983). An Apple II microcomputer for real-time control in a behavioral laboratory. Behavior Research Methods \& Instrumentation, 15, 158-166.

Notterman, J. M., \& MinTz, D. E. (1965). Dynamics of response. New York: Wiley. 